\section{Sur les familles inductives et projectives d'ensembles ${ }^{1}$ ).}

Par

\section{W. Sierpiński (Varsovie).}

Nous considérerons des familles formées d'ensembles de points d'un espace euclidien à un nombre fini de dimensions, mais pas nécessairement le même pour tous les ensembles de la famille considérée.

Une famille $F^{\prime}$ d'ensembles sera dite inductive, si elle contient les intervalles (à un nombre fini quelconque de dimensions) et si elle contient les sommes et les produits d'une infinité dénombrable d'ensembles de $F$ (appartenant au même espace à un nombre fini quelconque de dimensions) ${ }^{2}$ ).

On voit sans peine que toute famille inductive contient la famiiie de tous les ensembles mesurables $B$ qui est la plus petite famille inductive.

Voici un autre exemple d'ane famille inductive: c'est la famille do tous les ensembles de lu forme $P X+Q$, où $X$ est un ensemble donné (d'ailleurs quelconque) et $P$ et $Q$ sont des ensembles mesurables $B$. On pourrait démontrer que c'est la plus petite famille inductive contenant l'ensemble $X^{3}$ ).

$F$ étant une famille donnée d'ensembles, nous désignerons par $C(F)$ la famille de tous les ensembles complémentaires anx ensem-

1) Présenté au Congrès Intern. des Math. à Bologne, le 5 septembro 1928.

2) Cf. la définition de la propriété inductive de M. N. Lasin: Fundamenta Mathematicae t. X, p. 38.

s) M. Taraki a prouvé résemment qu'il existe $2^{22^{2 N_{0}}}$ familles inductives d'ensembles de points: sa démonstration paraitra dans lo vol. XIV do ce journal. bles de la famille $F$ (le complémentaire de chacun ensemble pris par rapport à l'espace dans lequel il est situé). On voit sans peine que Si la famille $F$ est inductive, la famille $C(F)$ l'est aussi.

En effet, si la famille $F$ est inductive, tout ensemble mesurable $B$ appartient à $F$, donc aussi à $C(F)$. Done $C(F)$ contient tous les intervalles (en tant que mesurables $B$ ). Pour prouver que la famille $C(F)$ est inductive il suffit de se rapporter encore aux formules

ei

$$
C\left(E_{1}+E_{2}+E_{3}+\ldots\right)=C E_{1} \cdot C E_{2} \cdot C E_{3} \ldots
$$

$$
C\left(E_{1}^{\prime} E_{2} E_{3}^{\prime} \ldots\right)=C E_{1} \cdot 1 \cdot C E_{2}^{\prime}+C E_{8}+\ldots
$$

où $C E$ désigne le complémentaire de l'ensemble $E$ ).

Nous désignerons par $R_{m}$ l'espace euclidien à $m$ dimensions, c'est-à-dire l'ensemble de tous les systèmes $\left(x_{1}, x_{2}, \ldots, x_{m}\right)$ de $m$ nombres réels $x_{1}, x_{2}, \ldots, x_{\mathrm{m}}$.

$E$ étant un ensemble situé dans $R_{m}$, nous appellerons projection de $E$ (sur $R_{m-1}$ ) et désignerons par $P(E)$ l'ensemble de tous les points $\left(x_{1}, x_{2}, \ldots, x_{m-1}\right)$ (de $R_{m-1}$ ) pour lesquels il existe un nombre réel $x$, tel que le point $\left(x_{1}, x_{2}, \ldots, x_{m-1}, x\right)$ appartient à $E$.

On voit sans peine que

$$
P\left(E_{1}+E_{2}+E_{3}+\ldots\right)=P\left(E_{1}\right)+P\left(E_{2}\right)+P\left(E_{3}\right)+\ldots
$$

Au lieu de $P P(E)$ nous écrirons $P^{\mathbf{s}}(E)$, et, généralement, nous poserons $P^{n}(E)=P\left(P^{n-1}(E)\right)$, pour $n=2,3, \ldots$

$F$ étant une famille donnée d'ensembles, nous désignerons par $P(F)$ la famille de tous les ensembles $P(E)$, où $E \in F$.

$E$ étant un ensemble situé dans $R_{\mathrm{m}}$, nous désignerons par $Q(E)$ l'ensemble de tous les points (de $\left.R_{m+1}\right)\left(x, x_{1}, x_{2}, \ldots, x_{m}\right)$, tels que $\left(x_{1}, x_{2}, \ldots, x_{m}\right) \in E$.

$F$ étant une famille donnée d'ensembles, nous désignerons par $Q(F)$ la famille de tous les ensembles $Q(E)$, où $E \in F$.

On roit sans peine que

$$
Q\left(E_{1}+E_{2}+E_{8}+\ldots\right)=Q\left(E_{1}\right)+Q\left(E_{2}\right)+Q\left(E_{3}\right)+\ldots
$$

et que

$$
C(Q(E))=Q(C(E))
$$

On voit aussi sans peine qu'on a toujours

$$
P(Q(E))=Q(P(E)) .
$$


Une famille $F$ d'ensembles sera dite projective, si

$$
P(F) \subset F \text { et } Q(F) \subset F .
$$

La plus petite famille inductive et projective d'ensembles est la famille de tous les ensembles $(A)$ (analytiques) de MM. Souslin et Lusin.

Si $F$ est une famille projective, $C\left(F^{\prime}\right)$ peut ne l'être pas, p. e. si $F$ est la famille de tous les ensembles $(A)$. Or, on a le suivant

Théorème. Si $F$ est une famille inductive et projective, la famille $P C(F)$ est aussi inductive et projective.

C'est la démonstration de ce théorème (dont les conséquences sont importantes pour la théorie des ensembles projectifs) qui est le but de ce Mémoire.

Lemme I. $R_{m}$ est une image continue de l'ensemble de tous les nombres irrationnels de l'intervalle $(0,1)$.

Dém. $\left.{ }^{1}\right)$ L'ensemble $H_{m}$ de tous les points de $R_{m}$, dont les coordonnées sont toutes irrationnelles, est, comme on sait, homéomorphe à l'ensemble $M_{0}$ de tous les nombres irrationnels de l'intervalle $\left(0, \frac{1}{2^{m}}\right)$ : soient

$$
x_{1}=\theta_{1}(t), \quad x_{2}=\theta_{2}(t), \ldots, \quad x_{m}=\theta_{m}(t)
$$

les fonctions définies et continues dans $M_{0}$, établissant cette homéomorphie.

Soient maintenant $k$ un entier, tel que $0 \leqslant k<2^{m}$,

(1) $k=\left(\alpha_{1} \alpha_{2} \ldots \alpha_{m}\right)_{2}=\alpha_{1} \cdot 2^{m-1}+\alpha_{2} \cdot 2^{m-2}+\ldots+\alpha_{m-1} \cdot 2+\alpha_{m}$

- son développement dyadique.

Désignons par $M_{k}$ l'ensemble de tous les nombres irrationnels de l'intervalle $\left(\frac{k}{2^{m}}, \frac{k+1}{2^{m}}\right)$, et posons, pour $t \in M_{k}$ :

(2)

$$
\left\{\begin{array}{c}
x_{1}=\alpha_{1} \sqrt{2}+\theta_{i}\left(t-\frac{k}{2^{m}}\right), \quad x_{2}=a_{2} \sqrt{2}+\theta_{2}\left(t-\frac{k}{2^{m}}\right), \ldots \\
x_{m}=\alpha_{m} \sqrt{2}+\theta_{m}\left(t-\frac{k}{2^{m}}\right)
\end{array}\right.
$$

1) La démonstration de ce lemme pourrait être un peu abrégée, si l'on utiliserait la courbe continue de M. P e a no remplissant $R_{m}$. Il résulte d'ailleurs immédiatement de li théorie des ensernbles analytiques.
Désignons par $T_{k}$ l'ensemble de tous les nombres (2) pour $t \in M_{k}$ : on voit sans peine que

$$
R_{m}=T_{0}+T_{1}+T_{2}+\ldots+T_{2^{m}-1} .
$$

En effet, soit $\left(x_{1}, x_{2}, \ldots, x_{m}\right)$ un point de $R_{m}$. Désignons, pour $i=1,2, \ldots, m$, par $\alpha_{i}$ le nombre 1 ou 0 , selon que la coordonnée $x_{i}$ est rationnelle ou non.

Les nombres

$$
x_{i}-\alpha_{i} / \overline{2} \quad(i=1,2, \ldots, m)
$$

sont done tous irrationnels et parsuite il existe un nombre $\tau$ de $M_{0}$, tel que

$$
x_{i}-\alpha_{i} \sqrt{2}=\theta_{i}(\tau), \text { pour } i=1,2, \ldots, m .
$$

Or, désignons par $k$ le nombre (1), et posons:

$$
t=\frac{k}{2^{m}}+\tau \text { : }
$$

$\tau$ ètant un nombre de $M_{0}, t$ appartiendra évidemment à $M_{k}$ et, d'après (4) et (5), nous aurons les formules (2), ce qui prouve (vu la définition de $\left.T_{k}\right)$ que le point $\left(x_{1}, x_{2}, \ldots, x_{m}\right)$ appartient à $T_{k}$ : la formule (3) est ainsi établie.

$M=M_{0}+M_{1}+\ldots+M_{2^{m-1}}$ est évidemment l'ensemble de tous les nombres irrationnels de lintervalle $(0,1)$.

Soit $t$ un nombre de $M$ : il existe donc un entier (1) bien déterminé (par $t)$, tel que

$$
\frac{k}{2^{m}}<t<\frac{k+1}{2^{m}}, \text { et } t \in M M_{k} .
$$

Posons

$$
f_{i}(t)=\alpha_{i} / \sqrt{2}+\theta\left(t-\frac{k}{2^{m}}\right), \text { pour } i=1,2,3, \ldots
$$

Les fonctions $f_{i}(t)(i=1,2, \ldots, m)$ seront évidemment définies et continues dans $M I$ et (d'après la définition de $T_{k}$ ) l'ensemble de tous les points

$$
\left(f_{1}(t), f_{2}(t), \ldots, f_{m}(t)\right), \text { pour } t \in M L_{k}
$$

sera évidemment l'ensemble $T_{k}$, done, d'après (3), l'ensemble de tous les points

$$
\left(f_{1}(t), f_{2}(t), \ldots, f_{m}(t)\right), \text { pour } t \in M
$$

sera l'ensemble $R_{m}$. 
Le lemme I est ainsi démontré.

Lemme II. Si $F$ est une famille inductive et projective, tout ensemble de la famille $C\left(F^{\prime}\right)$ est une image continue d'un ensemble de nombres irrationnels de l'intervaile $(0,1)$ appartenant à $C(F)$.

Dém. Soit $E$ un ensemble de la famille $C(F)$, situé dans $R_{m}$, et désignons par $H$ l'ensemble de tous les nombres $t$ de $M$, pour lesquels le point

$$
\left(f_{1}(t), f_{2}(t), \ldots, f_{m}(t)\right)
$$

appartient à $E^{\prime}\left(M\right.$ et $f_{i}(t)$ ayant les mêmes significations que dans la démonstration du lemme I). Je dis que $H$ appartient à $C(F)$.

Désignons par $S$ l'ensemble de tous les points

$$
\left(t, f_{1}(t), f_{2}(t), \ldots, f_{m}(t)\right)
$$

de $R_{m+1}$, où $t \in M$. Les fonctions $f_{1}(t)(i=1,2, \ldots, m)$ étant continues dans l'ensemble $M$ (qui est un $G_{\delta}$ ), on voit sans peine que l'ensemble $S$ est mesurable $B$ (un $G_{j}$ ).

D'après $E_{\epsilon} C(F)$, nous avons $C E \in F$, done, la famille $F^{\prime}$ étant projective, $Q(C E) \in F$. Or, $S$ étant mesurable $B$, on a $S \in F$, donc, d'après $Q(C E) \epsilon F$, la famille $F$ étant inductive, nous trouvons $S . Q(C E) \in F$, et parsuite (la famille $F$ étant projective)

6)

Or, je dis que

$$
P^{m}[S \cdot Q(C E)] \in F \text {. }
$$

7)

$$
M-H=P^{m}[S, Q(C E)] .
$$

En effet, soit $t_{0} \in M-H$. D'après la définition de $H$, nous avons

$$
\left(f_{1}\left(t_{0}\right), f_{2}\left(t_{0}\right), \ldots, f_{m}\left(t_{0}\right)\right) \in C E
$$

or, $t_{0} \in M$, donc, d'après la définition de $S$ :

(9)

$$
\left(t_{0}, f_{1}\left(t_{0}\right), f_{\mathbf{8}}\left(t_{0}\right), \ldots, f_{m}\left(t_{0}\right)\right) \in S .
$$

Or, d'après $(8)$ et la définition de l'opération $Q$, nous avons. douc, d'après (9):

$$
\left(t_{0}, f_{1}\left(t_{0}\right), f_{\mathbf{2}}\left(t_{0}\right), \ldots, f_{m}\left(t_{0}\right)\right) \in Q(C E)
$$

$$
\left(t_{0}, f_{1}\left(t_{0}\right), f_{2}\left(t_{0}\right), \ldots, f_{m}\left(t_{0}\right)\right) \in S \cdot Q(C E)
$$

et parsuite (d'après la définition de l'opération $P$ ):

$$
t_{0} \in P^{m}[S . Q(C E)]
$$

D'autre part, soit $t_{0} \in P^{m}[S . Q(C E)]$. Il existe donc un point $x_{1}, x_{2}, \ldots, x_{m}$ de $R_{m}$, tel que

$$
\left(t_{0}, x_{1}, x_{2}, \ldots, x_{m}\right) \in S . Q(C E) .
$$

D'après la définition de $S$, il en résulte que

et

$$
t_{0} \in M
$$

$$
x_{1}=f_{1}\left(t_{0}\right), \quad x_{2}=f_{2}\left(t_{0}\right), \ldots, \quad x_{m}=f_{m}\left(t_{0}\right)
$$

d'après $(10)$ et (12) nous trouvons

$$
\left(t_{0}, f_{1}\left(t_{0}\right), f_{2}\left(t_{0}\right), \ldots, f_{m}\left(t_{0}\right)\right) \in Q(C E)
$$

donc (d'après la définition de l'opération $Q$ ):

$$
\left(f_{1}\left(t_{0}\right), f_{9}\left(t_{0}\right), \ldots, f_{m}\left(t_{0}\right)\right) \in C E
$$

ce qui donne, vu la définition de l'ensemble $H$ :

donc, d'après $(11)$ :

$$
\begin{gathered}
t_{0} \in C H, \\
t_{0} \in M-H .
\end{gathered}
$$

La formule (7) est ainsi établie. Il en résulte, d'après (6), que $M-H \in F$, done

$$
C(M-H) \epsilon C F \text {. }
$$

Or, nous avons $H \subset M$, ce qui donne

$$
H=M . C(M-H) \text {. }
$$

La famille $C F$ étant inductive (puisque la famille $F$ l'est), et $M$ étant un ensemble mesurable $B$ (donc $\epsilon C(F)$ ), nous trouvons, d'après (13) et (14): $H_{\epsilon} C F$, c q. f. d.

Or, d'après la définition de l'ensemble $H$ (et des fonctions $f_{1}(t)$, $i=1,2, \ldots, m)$, l'ensemble $E$ est une image continue de l'ensemble $H$ (de nombres irrationnels appartenant à lintervalle $(0,1))$ : la formule $H_{\epsilon} C F$ prouve done le lemme II.

Lemme III. Si $F$ est une famille inductive et projective d'ensembles, toute image continue d'un ensemble de la famille $C(F)$ appartient $\grave{a}$ la famille $P C(F)$.

Dém. Soit $E$ un ensemble situé dans $R_{m}$ qui est une image continue d'un ensemble de la famille $C(F)$ (situé dans $R_{n}$ ). Du 
lemme II résulte tout de suite que $E$ est une image continue d'un ensemble linéaire appartenant à $C(F)$, soit $H$. Il existe done $n$ fonctions $\varphi_{i}(t) \quad(i=1,2, \ldots, m)$, définies et continues dans $H$ et telles que $E$ est l'ensemble de tous les points

$$
\left(\varphi_{1}(t), \varphi_{2}(t), \ldots, \varphi_{m}(t)\right) \text {, pour } t \in H .
$$

Désignons par $T$ l'ensemble de tous les points de $R_{m}$

$$
\left(\varphi_{1}(t), \varphi_{\mathbf{z}}(t), \ldots, \varphi_{m}(t), t\right), \quad \text { où } \quad t \in H
$$

je dis que l'ensemble $T$ appartient à $C(F)$.

Les fonctions $\varphi_{i}(t)(i=1,2, \ldots, m)$ étant continues dans $H$, on a, comme on roit sans peine:

$$
T=\bar{T} \cdot Q^{m}(H)
$$

où $\bar{T}$ désigne la fermeture de $T$ (cest-à-dire $T=T+T^{\prime}$ ), et où $Q^{k}(H)=Q\left(Q^{k-1}(H)\right)$, pour $k=2,3, \ldots, m$.

En effet, on a évidemment $T \subset \bar{T}$, ot, pour prouver la formule (15) il suffira de démontres que $\bar{T} \cdot Q^{m}(t i) \subset T$. Soit donc $p\left(x_{1}, x_{2}, \ldots, x_{m}, x_{m+1}\right)$ un point de l'en sermble $\bar{T} . Q^{m}(H)$. D'après $p \in Q^{m}(H)$, nous avons $x_{m+1} \in H$. Or, de $p \in \bar{T}$ résulte $p=\lim _{n \rightarrow \infty} p_{n}$, où $p_{n} \in T$, pour $n=1,2,3, \ldots$ Soit $p_{n}=\left(x_{1}^{(n)}, x_{2}^{(n)}, \ldots, x_{m+1}^{(n)}\right):$ d'après $p_{n} \in T$ et d'après la définition de $T$, nous avons $x_{m+1}^{(n)} \in H$ et $x_{i}^{(n)}=\varphi_{1}\left(x_{m+1}^{(n)}\right)$, pour $i=1,2, \ldots, m$. Or, de $p=\lim _{n=\infty} p_{n}$ résulte que $\lim _{n \rightarrow \infty} x_{m+1}^{(n)}=x_{m+1}$, donc, les fonctions $\varphi_{i}$ étant continues dans $H: \lim _{n \rightarrow \infty} x_{i}^{(n)}=\lim _{n \rightarrow \infty} \varphi_{i}\left(x_{m+1}^{(n)}\right)=\varphi_{i}\left(x_{m+1}\right)$, pour $i=1,2, \ldots, m$. D'autre part, on a, d'après $\lim _{n=\infty} p_{n}=p: \lim _{n \rightarrow \infty} x_{i}^{(n)}=x_{i}$. On a donc $x_{i}=\varphi_{i}\left(x_{m+1}\right)$ pour $i=1,2, \ldots, n$, ce qui proure (d'après $x_{m+1} \in H$ ) que $p \in T$, c. q. f. d.

Or, la famille $F$ étant projective, on a $Q(F) \subset F$, donc aussi $Q\left(C\left(F^{\prime}\right)\right) \subset C\left(F^{\prime}\right)$ et $Q^{\mathrm{m}}(C(F)) \subset C\left(F^{\prime}\right):$ l'ensemble $H$ appartenant à $C\left(F^{\prime}\right)$, il en résulte que $Q^{m}(H) \in C(F)$.

Or, la famille $C(F)$ est inductive (puisque la famille $F$ l'est): l'ensemble $\bar{T}$, comme fermé (donc mesurable $B$ ) appartient donc à $C(F)$, et la formule (15) prouve (d'après $\left.Q^{m}(H)_{\epsilon} C(F)\right)$ que $T_{\epsilon} C(F)$.

D'autre part, de la défnition de $T$ résulte sans peine que $P(T)=E$. Notre lemme III est ainsi démontré.

La projection d'un ensemble étant évidemment une image continue de cet ensemble, il résulte tout de suite de notre lemme ce
Corollaire. Si $F$ est une famille inductive et projective d'ensembles, on a:

$$
P P(C(F)) \subset P(C(F))
$$

Nous allons maintennant à démontrer notre théorème. Soit $F$ une famille inductive et projective. D'après le corollaire du lemme III on a done la formule (16).

Or, la famille $F$ étant projective, nous avons

$$
Q(E)_{\epsilon} F, \text { pour } E_{\epsilon} F
$$

ce qui donne tout de suite

$$
C Q(E){ }_{\epsilon} C(F) \text { pour } E \in F \text {, }
$$

donc, puisq'a'on a toujours $C Q(E)=Q(C E)$ :

$$
Q(C E)_{\epsilon} C(F) \text {, pour } E \in F \text {, }
$$

d'où

$$
P Q(C E) \in P C(F) \text {, pour } E \in F,
$$

et, puisque toujours $P Q(Z)=Q P(Z)$ :

$$
Q P(C E) \epsilon P C\left(F^{\prime}\right), \text { pour } E_{\epsilon} F,
$$

ce qui donne tout de suite:

$$
Q P(C(F)) \subset P C(F)
$$

Les formules (16) et (17) prouvent que la famille $P C\left(F^{\prime}\right)$ est projective.

Pour démontrer notre théorème, il nøus reste donc à prouver que la famille $P C(F)$ est inductive.

Soit $E$ un intervalle à $m$ dimensions, c'est-à-dire l'ensemble de tous les points $\left(x_{1}, x_{2}, \ldots, x_{m}\right)$ de $R_{m}$, tels que

$$
a_{i} \leqslant x_{i} \leqslant b_{i}, \text { pour } i=1,2, \ldots, m
$$

(où $a_{i}$ et $b_{i}, i=1,2, \ldots, m$, sont des nomires réels donnés).

Désignons par $H$ i'ensemble de tous les points $\left(x_{1}, x_{2}, \ldots, x_{m}, 0\right)$ de $R_{m+1}$, tels que $\left(x_{1}, x_{2}, \ldots, x_{m}\right) \in E$ : l'ensemble $H$ sera éridemment mesurable $B$, donc aussi $C H$. La famille $F$ étant inductive, il en résulte que $C H \epsilon F$, donc $H_{\epsilon} C(F)$, ce qui donne $P(H) \in P(C(F))$. Or, on a évidemment $P(H)=E$ : la formule $P(H) \in P(C(F))$ donne 
done $E \in P(C(F))$. Nous avons ainsi démontré que la famille $P\left(C\left(F^{\prime}\right)\right)$ contient tous les intervalles (à un nombre finc quelconque de dimensions).

Soit maintenant $E_{\mathrm{n}}(n=1,2,3, \ldots)$ une suite infinie d'ensembles de la famille $P C(F)$, et posons

$$
E=E_{1}+E_{2}+E_{3}+\ldots
$$

D'après $E_{n} \in P C\left(F^{\prime}\right)$, pour $n=1,2,3, \ldots$, nous pouvons poser

$$
E_{n}=P\left(C H_{n}\right), \quad \text { où } \quad H_{n} \in F, \text { pour } n=1,2,3, \ldots
$$

La famille $F$ étant inductive, il résulte de $H_{n} \in F$ (pour $n=1,2,3, \ldots$ ) que

donc

c'est-à-dire

$$
H_{1} H_{2} H_{3} \ldots \epsilon F^{\prime}
$$

d'où

$$
C\left(H_{1} H_{2} H_{3} \ldots\right) \in C(F),
$$$$
C H_{1}^{r}+C_{2}+C H_{3}+\ldots \epsilon C(F) \text {, }
$$

$P\left(\mathrm{CH}_{1}\right)+P\left(\mathrm{CH}_{2}\right)+P\left(\mathrm{CH}_{\mathrm{s}}\right)+\ldots=P\left(\mathrm{CH}_{1}+\mathrm{CH}_{\mathbf{2}}+\ldots\right) \epsilon P C(F)_{\text {, }}$

c'est-à-dire, d'après $(18)$ et $(19), E \in P C(F)$.

Nous avons ainsi démontré que la famille $P C(F)$ contient les sommes d'une infinité dénombrable d'ensembles de $P C\left(F^{\prime}\right)$.

Soit maintenant $E_{n}(n=1,2,3, \ldots)$ une suite inflnie d'ensembles. de la famille $P C(F)$, et posons

$$
E=E_{1} E_{\mathbf{9}} E_{\mathbf{3}} \ldots
$$

Soit $n$ un indice donné. D'après $E_{n} \in P C(F)$ et d'après le lemme II, $E_{n}$ est une image continue d'un ensemble $X_{n}$ de nombres irrationnels de l'intervalle $(0,1)$, appartenant à $C\left(F^{\prime}\right)$. Il existe donc une fonction $f_{n}(x)$, définie et continue dans $X_{n}$, telle que

$$
E_{n}=f_{n}\left(X_{n}\right) \text {. }
$$

Soit $x$ un nombre irrationnel de lintervalle $(0,1)$,

$$
x=\frac{1}{\nu(1, x)}+\frac{1}{\mid \nu(2, x)}+\frac{1}{\mid \nu(3, x)}+\ldots
$$

- son déreloppement en fraction continue. Posons (pour $n=1,2,3, \ldots$ ):

(23) $\varphi_{x}(x)=\frac{1}{\nu\left(1.2^{n-1}, x\right)}+\frac{1}{\mid \nu\left(3.2^{n-1}, x\right)}+\frac{1}{\mid \nu\left(5.2^{n-1}, x\right)}+\ldots$
Les fonctions $\varphi_{n}(x)$ seront évidemment définies et continues dans l'ensemble $N$ de tous les nombres irratiounels de l'intervalle $(0,1)$.

Désignons par $N_{n}$ l'ensemble de tous les nombres $x$ de $N$, tels que $\varphi_{n}(x) \in X_{a}$. Je dis que $N_{n} \in C(F)$.

En effet, d'après $X_{n} \in C(F)$, nous avons $C X_{n} \in F$, done, la famille $F$ étant projective, $Q\left(C X_{n}\right) \in F$. Or, désignons par $\Gamma$ l'ensemble de tous les points $(x, y)$ du plan, tels que

$$
x \in N, \text { et } y=\varphi_{n}(x) \text {; }
$$

la fonction $\varphi_{n}(x)$ étant continue dans $N$, on roit sans peine que l'ensemble $F$ est un $G_{\delta}$, donc (la famille $F$ étant inductive): $\Gamma \in F$.

Or, nous avons la formule

$$
N-N_{n}=P\left[Q\left(C X_{n}\right) \cdot I\right] .
$$

En effet, si $x_{0} \in N-N_{n}$, on a $x_{0} \in N$ et $x_{0}$ non $\in N_{n}$, donc (d'après la définition de $\left.N_{n}\right): \varphi_{n}\left(x_{0}\right)$ non $\epsilon X_{n}$, c'est-à-dire $\varphi_{n}\left(x_{0}\right) \epsilon C X_{n}$, ce qui donne (vu la définition de l'opération $Q):\left(x_{0}, \varphi_{n}\left(x_{0}\right)\right) \in Q\left(C X_{n}\right)$, et (vu la définition de $\Gamma$ ): $\left(x_{0}, \varphi_{n}\left(x_{0}\right)\right) \in \Gamma$, donc $\left(x_{0}, \varphi_{x}\left(x_{0}\right)\right) \in Q\left(C X_{n}\right) . T$, et parsuite $x_{0} \in P\left[Q\left(C X_{n}\right) . \Gamma\right]$.

D'autre part, soit $x_{0} \in P\left[Q\left(C X_{n}\right) . \Gamma\right]$. Il existe done un nombre réel $y_{0}$, tel que $\left(x_{0}, y_{0}\right) \in Q\left(C X_{n}\right) . \Gamma$, donc $\left(x_{0}, y_{0}\right) \in Q\left(C X_{n}\right)$, ce qui donne $y_{0} \in C X_{n}$, et $\left(x_{0}, y_{0}\right) \in T$, ce qui donne (vu la définition de $I$ ) $x_{0} \in N$ et $y_{0}=\varphi\left(x_{0}\right)$.

Nous avons donc (d'après $\left.y_{0} \in C X_{n}\right): \varphi_{n}\left(x_{0}\right) \in C X_{n}$, donc (d'après la déflnition de $N_{n}$ ): $x_{0}$ non $\epsilon N_{n}$, ce qui donne, d'après $x_{0} \in N$, $x_{0} \in N-N_{n}$.

La formule (24) ost ainsi établie.

D'après $Q\left(C X_{n}\right) \in F$ et $T \in F$, la famille $F$ étant inductive et projective, on a $P\left[Q\left(C X_{n}\right), \Gamma\right] \in F$ : la formule (24) proure done que

Or, d'après $N_{n} \subset N$, on a éridemment

$$
N_{n}=N . C\left(N-N_{n}\right) \text {. }
$$

La famille $F$ étant inductive, on a $N_{\epsilon} C(F)$, donc, d'après (25), la formule (26) donne (la famille $C(F)$ étant inductive en tant que $F): N_{n} \in C(F)$, c. q. f. d. $\begin{aligned} & \text { Posons } \\ & \text { P) }\end{aligned} N_{0}=N_{1} N_{2} N_{3} \ldots$; 
la famille $F$, et parsuite aussi $C(F)$, étant inductive, et les ensembles $N_{n}(n=1,2,3, \ldots)$ appartenant $\hat{a} C(F)$, la formule (27) prouve que $N_{0} \in C(F)$.

Désignons par $X_{0}$ l'ensemble de tous les nombres $x$ de $N_{0}$, tels que

$$
f_{n}\left(\varphi_{n}(x)\right)=f_{1}\left(\varphi_{1}(x)\right), \text { pour } n=1,2,3, \ldots ;
$$

nous aurons donc

$$
f_{n}\left(\varphi_{n}\left(X_{0}\right)\right)=f_{1}\left(\varphi_{1}\left(X_{0}\right)\right), \text { pour } n=1,2,3, \ldots
$$

Les fonctions $f_{n}\left(\varphi_{n}(x)\right)$ étant continues dans l'eusemble $N_{0}$, on voit sans peine que l'ensemble $X_{0}$ est fermé dans $N_{0}$. Donc, $X_{0}=$ $=N_{0}$. $\Phi$, où $\Phi$ est un ensemble fermé, donc $\Phi \in C(F)$, ce qui donne, d'après $N_{0} \in C(F), X_{0} \in C(F)$.

On a maintenant la formule

$$
f_{1}\left(\varphi_{1}\left(X_{0}\right)\right)=E
$$

pour la démontrer, il suffit de répéter mot-à-mot la démonstration de cette égalité que j'ai donné dans le volume XI de ce journal, p. 124 et 125 , formule (5).

L'ensemble $X_{0}$ appartenant a $C(F)$ et la fonction $f_{1}\left(\varphi_{1}(x)\right)$ étant continue dans $X_{0}$, la formule (29) prouve que l'ensemble $E$ est une image continue d'un ensemble de la famille $C(F)$. D'après le lemme III, l'ensemble $E$ appartient donc à la famille $P C\left(F^{\prime}\right)$.

Nous avons ainsi démontré que la famille $P C\left(F^{T}\right)$ contient les produits d'une infinité dénombrable d'ensembles do $P C(F)$.

Il est ainsi établi que la famille $P C(F)$ est inductive. Or, plus hant nous avons démontré qu'elle est projective. Notre théorème est ainsi démontré complètement.

Voici une application de notre théorème à la théorie des ensembles projectifs de M. N. Lusin.

$\mathcal{F}_{0}$ désignant la famille de tous les ensembles fermés, posons

(30) $\mathscr{d}_{0}=P\left(\tilde{\mathcal{O}}_{0}\right)$, et $\mathcal{C}_{n-1}=C\left(\mathscr{\mathscr { d }}_{n-1}\right), P_{n}=P\left(\mathcal{C}_{n-1}\right)$, pour $n=1,2,3, \ldots$

Les ensembles appartenant aux familles $\mathscr{J}_{n}$ et $\mathcal{C}_{n}$ sont appelés resp. $P_{n}$ et $\left.C_{n}{ }^{1}\right)$ : ils coïncident avec les ensembles projectifs de classe $\leqslant n$ de M. Lusin ${ }^{2}$ ).

1) Voir p. e. Fund. Math. t. Xl, p. 121-122.

2) Fund. Math. t. $\mathrm{X}$, p. 90.
Les ensembles $P_{0}$ coïncident avec les ensembles $F_{\sigma}{ }^{1}$ ), donc les ensembles $C_{0}-$ avec les $G_{\delta}$, et parsuite les ensembles $P_{1}-$ avec les ensembles $(A)$ (analytiques)." Or, la famille $\mathscr{D}_{1}$ des ensembles $(A)$ est, comme on sait, inductive et projective ${ }^{2}$ ).

soit $n$ un nombre naturel, et admettons que la famille $\mathscr{P}_{n}$ est inductive ot projective (ce qui est vrai pour $n=1$ ). D'après (30), nous avons

$$
\mathscr{P}_{n+1}=P C\left(\mathscr{P}_{n}\right)
$$

et. en vertu de notre théorème, nous concluons que la famille $\mathscr{A}_{n+1}$ est inductive es projective.

Il en résulte done, par linduction, que les familles $\mathscr{P}_{n}$ sont inductives et projectives pour $n=1,2,3, \ldots$ Donc d'après (1), les tamilles $\mathfrak{e}_{n}(n=1.2,3, \ldots)$ sont inductives (elles ne sont pas, d'ailleurs, projectives). Nous obtenons ainsi les prupositions snivantes:

Toute somme th tout produit d'une infinité d'nombrable d'ensembles $P_{n}$ est un exsemble $P_{n}$ (pour $\left.n=1,2,3, \ldots\right)^{3}$ ).

Tuute somme et tout produit d'une infinité dénombrable d'ensembles $C_{n}$ est uni ensemble $C_{n}$ (pour $n=1,2,3, \ldots$ ).

1) Voir Fund. Math. t. VII, p. 238.

3) Fund. Math. t. X, p. 38 et 17.

3) Nous avons déjà signalé ce théorème dans le vol. Xl de ce journal, p. 126. Yoir aussi ma Note dans les C. R. t. 185, p. 834 (séance du $2 \dot{4}$ octobre 1927). 